\title{
Salmonella Typhimurium resides largely as an extracellular pathogen in porcine tonsils, independently of biofilm-associated genes $\operatorname{csg} A, \operatorname{csg} D$ and $a d r A$
}

\author{
Alexander VAN PARYS ${ }^{a}$, Filip BOYEN ${ }^{a}$, Jiri VOLF ${ }^{b}$, Elin VERBRUGGHE ${ }^{a}$, Bregje LEYMAN ${ }^{a}$, Ivan \\ RYCHLIK $^{b}$, Freddy HAESEBROUCK ${ }^{a}$ and Frank PASMANS ${ }^{a}$ \\ ${ }^{a}$ Department of Pathology, Bacteriology and Avian Diseases, Faculty of Veterinary Medicine, \\ Ghent University, Merelbeke, Belgium \\ ${ }^{b}$ Veterinary Research Institute, Hudcova, Brno, Czech Republic
}

\section{INTRODUCTION}

In European countries, Salmonella enterica subspecies enterica serovar Typhimurium (Salmonella Typhimurium) is the serovar most frequently isolated from slaughter pigs ${ }^{1}$. Porcine carcass contamination with Salmonella Typhimurium can largely be attributed to persistently infected pigs. Even though tonsils are a predilection site for Salmonella persistence in pigs, virulence mechanisms necessary for cell invasion and intracellular survival do not contribute to tonsillar colonization ${ }^{2}$, suggesting that Salmonella Typhimurium resides mainly extracellularly in porcine tonsils. Biofilm formation is a mechanism used by several bacteria to survive in an extracellular context or in hostile environments ${ }^{3}$. The role of biofilm formation in Salmonella Typhimurium persistence in pigs is still unknown. It was the aim of the present study to determine whether Salmonella Typhimurium persists intracellularly or extracellularly in tonsils of pigs. Additionally, the role of biofilm formation in persistence of Salmonella Typhimurium in porcine tonsils was determined.

\section{METHODS AND MATERIALS}

As wild type (WT), we used a virulent Salmonella Typhimurium phage type 120/Ad strain, isolated from a pig stool sample. In the in vivo trials, a spontaneous nalidixic acid resistant derivative of the wild type was used. Chloramphenicol-resistant substitution mutants in biofilm-associated genes $\operatorname{csg} A$, $\operatorname{csg} D$ and $a d r A$ were created as described by Datsenko and Wanner ${ }^{4}$. The difference in colony morphology between the WT and the mutants was examined by growing the strains on LB agar without salt, supplemented with Coomassie Brilliant Blue and Congo Red (Sigma-Aldrich) at $28{ }^{\circ} \mathrm{C}$ for 7 consecutive days. To determine the localization of Salmonella Typhimurium in porcine tonsils, 12 piglets were inoculated with $2 \times 10^{7}$ colony forming units (CFU) of the WT. Five and 28 days post inoculation, 8 and 4 pigs were euthanized respectively. Samples of tonsils were collected to prepare single cell suspensions (SCS) and cryosections. To obtain SCS, tonsils were rinsed, cut to small pieces and treated with $100 \mathrm{U} / \mathrm{ml}$ collagenase from Clostridium histolyticum Type V (Sigma-Aldrich). Half of the SCS was treated with $2 \%$ Triton X100 (Sigma-Aldrich) to lyse cells. These suspensions were then plated on BGA to assess the total number of intra- and extracellular bacteria. The remainder of SCS was treated with $100 \mu \mathrm{g} / \mathrm{ml}$ gentamicin (Sigma-Aldrich) to kill all extracellular bacteria. Cells were then washed and lysed with $2 \%$ Triton X-100. Ten-fold dilutions were plated on BGA to determine the number of intracellular bacteria. Samples that were negative at direct plating were enriched in tetrathionate broth (Merck). Finally, the percentage of extracellular bacteria was calculated. For cryosections, liquid nitrogen frozen tonsil samples were sectioned at $8 \mu \mathrm{m}$ with a cryotome, dried and fixed in acetone. Sections were preincubated with $30 \%$ goat serum to reduce background staining. Sections were subsequently incubated with a primary antibody mixture containing a polyclonal rabbit antibody targeting the Salmonella O4 somatic antigen (1:100 dilution; Pro Lab Diagnostics) and a mouse monoclonal antibody targeting CD172 $\alpha$ (1:50 dilution; AbD Serotec). After washing, the sections 
were incubated with a secondary antibody mixture containing goat anti-rabbit Alexa Fluor 488 and goat anti-mouse Alexa Fluor 568 (both 1:200 dilution; Molecular Probes). Sections were evaluated using a Leica DMRB fluorescence microscope (Leica Inc). Pictures were taken using a Moticam 2300 camera (Moticam Instruments Inc) and 100 bacteria in each tonsil sample were visually counted.

To examine the role of biofilm formation in Salmonella persistence in porcine tonsils, 3 groups of 6 piglets were orally inoculated with $2 \times 10^{7} \mathrm{CFU}$ of WT Salmonella and $2 \times$ $10^{7}$ CFU of $\Delta \operatorname{csg} A, \Delta \operatorname{csg} D$ or $\triangle a d r A$ Salmonella. Ten days after oral inoculation, pigs were euthanized and tonsils, ileum and ileum contents, ileocecal lymph nodes and faeces were collected. Tissue samples were cut to small pieces and stomached. Ten-fold dilutions of tissue samples, ileum contents and faeces were plated on BGA with nalidixic acid for selective growth of the WT and on BGA with chloramphenicol for selective growth of the substitution mutants. When negative at direct plating, samples were enriched in tetrathionate broth and again plated on BGA with the respective antibiotics.

\section{RESULTS}

After 7 days of incubation at $28^{\circ} \mathrm{C}$, the WT showed a biofilm-associated rough and dry morphotype, while the mutants exhibited a biofilm deficient smooth and white morphotype. Five and 28 days post inoculation, $97.0 \pm 2.0 \%$ and $97.6 \pm 3.6 \%$ of the Salmonella bacteria respectively was found to reside extracellularly in the tonsils using the single cell suspension method. These results were confirmed using immunohistochemistry. In the mixed infection experiment, in none of the analyzed samples of all three groups the $\operatorname{csg} A, \operatorname{csg} D$ or adrA Salmonella mutants were significantly impaired in colonization and persistence capacity compared to the wild type strain in any of the organs examined (FIGURE 1).

\section{DISCUSSION}

Previous work using a SPI-1 deficient strain suggested that Salmonella predominantly resided extracellularly in porcine tonsils ${ }^{2}$. In the present study we confirmed this extracellular localization using single cell suspensions and immunohistochemistry. In a subsequent mixed infection experiment however, we were unable to show a role for the biofilm-associated genes $\operatorname{csg} A, \operatorname{csg} D$ or $a d r A$ in this extracellular persistence ${ }^{5}$.

\section{REFERENCES}

${ }^{1}$ Anonymous (2008). Report of the task force on zoonoses data collection on the analysis of the baseline survey on the prevalence of Salmonella in slaughter pigs, in the EU, 2006-2007. Part A: Salmonella prevalence estimates. The EFSA Journal 135, 1-111.

${ }^{2}$ Boyen F, Pasmans F, Van Immerseel F, Morgan E, Adriaensen C, Hernalsteens JP, Decostere A, Ducatelle R and Haesebrouck F (2006). Salmonella Typhimurium SPI-1 genes promote intestinal but not tonsillar colonization in pigs. Microbes Infect. 8, 2899-2907.

${ }^{3}$ Rosenberg LE, Carbone AL, Römling U, Uhrich KE and Chikindas ML (2008). Salicylic acid-based poly(anhydride esters) for control of biofilm formation in Salmonella enterica serovar Typhimurium. Lett. Appl. Microbiol. 46, 593-599.

${ }^{4}$ Datsenko KA and Wanner BL (2000). One-step inactivation of chromosomal genes in Escherichia coli K-12 using PCR products. PNAS 97: 6640-6645. ${ }^{5}$ Van Parys A, Boyen F, Volf J, Verbrugghe E, Leyman B, Rychlik I, Haesebrouck F and Pasmans F (2010). Salmonella Typhimurium resides largely as an extracellular pathogen in porcine tonsils, independently of biofilm-associated genes $\operatorname{csg} A, \operatorname{csg} D$ and $a d r A$. Vet. Microbiol., In Press.

\section{TABLES AND FIGURES}

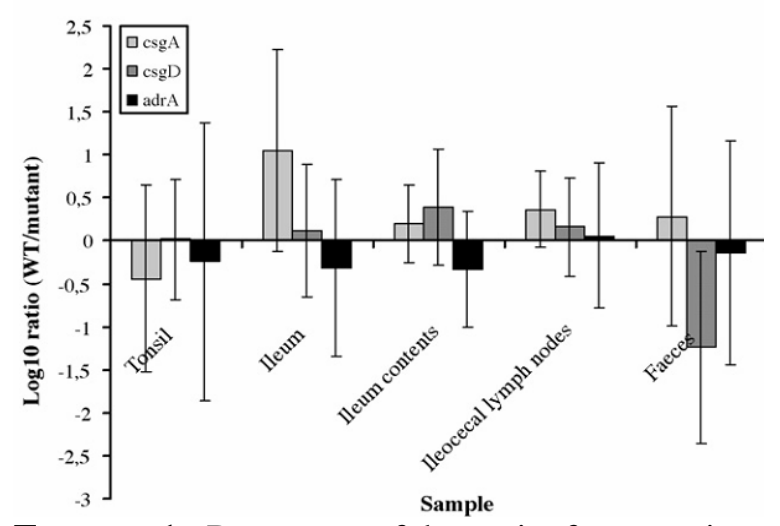

FIGURE 1. Recovery of bacteria from various organs of six piglets orally inoculated with a mixture of the wild type Salmonella Typhimurium and $\operatorname{csg} A, \quad \operatorname{csg} D$ or $a d r A$ Salmonella Typhimurium mutants. The $\log 10$ value of the ratio of CFU per gram sample of the wild type and the biofilm-associated gene mutants is given as the mean $\pm \mathrm{SD}$. 